\title{
Uncertainty of measurement - Important parameter for product conformity assessment
}

\author{
Gina-Mihaela SICOE \\ University of Pitești, Romania \\ *Corresponding author e-mail: gina_boicea@yahoo.com \\ Article history \\ Received 15.06.2020 \\ Accepted 20.09.2020 \\ DOI https://doi.org/10.26825/bup.ar.2020.008
}

\begin{abstract}
The measurements are widely used to ensure the quality of products. It is known that the measurement process is influenced by many factors which lead to measurement errors. The uncertainty of measurement is an essential parameter because the expression of the results accompanied by uncertainty offers more confidence in the results. The research presented in this article focuses on methods for calculating uncertainty and explaining the rules for making decisions when we use uncertainty. Two case studies are presented, one for dimensional measurements and the other for the estimation of the uncertainty of Vickers hardness measurement for one material, steel OLC15, in order to establish the relevance of the results and quality assessment. The results obtained on several measurements and the uncertainties of measurement associated are presented in the article and a discussion of the results is undertaken.
\end{abstract}

Key words: uncertainty of measurement, error, conformity

\section{Introduction}

In the production activity, the aim is to obtain a high level of quality, the objective being 'zero complaints from customers' or zero non-compliant products.

One method used on a large scale for control is represented by dimensional measurements. In many situations $100 \%$ control is applied. But, despite this type of control, it has been reported that nonconforming parts are recorded, which means that errors occur in the measurement process, even after $100 \%$ verification of the products.

In this article we will deal with the sources of these errors and how to express / interpret the results for a high degree of confidence. The analysis of some statistical data showed that applying different measurement methods on the same measurand, performing the measurements with different operators or using different measuring equipment, different results were obtained.

To determine the value of a characteristic, the question arises how to choose the right results from all the results obtained and how to estimate the errors that could occur.

The variability of the results is due to several factors:

- the measurements are subject to environmental conditions, temperature, humidity, pressure, etc., which influence the results obtained;

- the dimensions of a metal part may be influenced by its positioning;

- the measuring instrument is a source of error (response time, accuracy, sensitivity); 
- the operator never performs the same measurement under identical conditions (fatigue occurs, parallax errors);

- a measurement generally comprises several operations, each of them may be a source of variability.

By measurement, we mean a set of operations aimed to determining the value of a characteristic.

The "true value" of a measurand is the result that would have been obtained by perfect measurement.

\section{The concept of uncertainty}

ISO / IEC 17025 "General requirements for the competence of testing and calibration laboratories" and ISO / IEC guide Guide 98-3 (GUM) "ISO/IEC GUIDE 98-3, Uncertainty of measurement - Part 3: Guide to the expression of uncertainty in measurement" are the basic documents for the implementation and interpretation of measurement uncertainty.

The uncertainty of measurement represents a quantitative indicator of the quality of the results obtained from the measurements. Uncertainty of measurement is defined by standards and regulations and is a requirement for the certification of metrology or testing laboratories. In practice, knowing the uncertainty is very useful to make decisions about production quality when the measurement results are at the limits of the compliance area.

The word uncertainty means doubt; the uncertainty of the result of a measurement reflects the impossibility of knowing the exact value of the measurand. In this probabilistic view of error, the concept of uncertainty is defined as follows:

- uncertainty is a parameter associated with the result of a measurement, which characterizes the total dispersion of values that could reasonably be assigned to the measurement;

- uncertainty is an estimation characterizing the range of values in which the true value of a measured quantity lies.

The components of uncertainty are determined based on repeated measurements, previous measurements, knowledge of the equipment and experience of the operator. Uncertainty estimation involves an assessment of possible sources of uncertainty and a good knowledge of the measurement procedure. To establish the factors that influence the measurement uncertainty, we start from the $5 \mathrm{M}$ cause-effect diagram, fig. 1. [1]

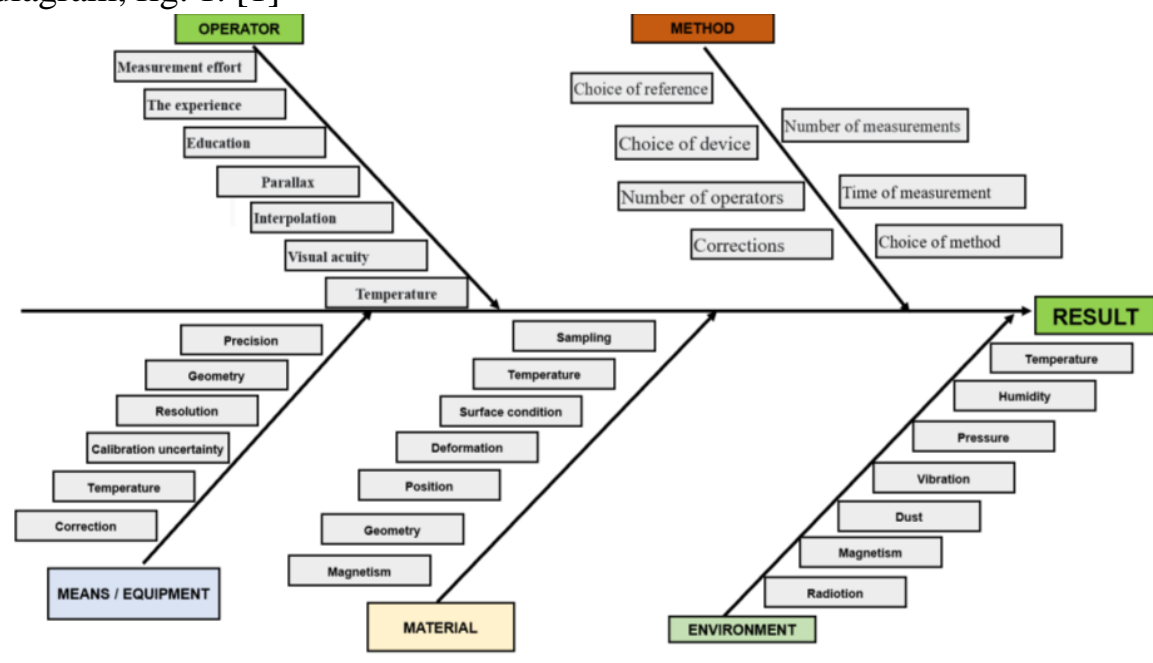

Figure 1. 5M Cause-effect diagram

\section{Calculation of uncertainty}

Uncertainty of repeatability, $u_{\text {repet, }}$ involves performing several measurements, $n$, preferably minimal $10: x_{1}, x_{2}, \ldots, x_{n}$ 
1. The repeatability uncertainty is calculated by the formula:

$$
u_{\text {repet }}=\frac{s_{i}}{\sqrt{n}} \quad s_{i}=\sqrt{\frac{\sum_{i=1}^{n}\left(x_{i}-\bar{x}\right)^{2}}{(n-1)}}
$$

Where:

- $\quad x_{i}$ is the value of the measurement;

- $\quad n$ is the number of measurements;

- $\overline{\boldsymbol{x}}$ is the average value,

- $\quad s_{i}$ is the standard deviation.

2. Uncertainty of reproducibility $u_{r e p r}$ is calculated by the formula:

$$
u_{\text {repr }}=\sqrt{\frac{1}{p-1} \sum_{i=1}^{1}\left(\overline{y_{i}}-y\right)^{2}+\frac{n-1}{n} \sum_{i=1}^{p} \frac{s_{i}^{2}}{p}}
$$

Where :

- $p$ is number of operators;

- $n$ is the number of repetitions per operator (minimum recommended 10);

- $\bar{y}_{\mathrm{i}}$ is the average of the measurements of an i operator;

$-\overline{\bar{y}}$ is the overall average of the measurements made by all operators.

3. Compound uncertainty. If the measurement is influenced by several sources of uncertainties (x), the compound uncertainty is calculated: $u_{c}(\mathrm{x})$ with the formula:

$$
u_{c}(x)=\sqrt{\sum_{i}\left(u_{i}(x)\right)^{2}}
$$

The measurement process associated with a manufacturing process aims to provide a measurement result associated with a declaration of conformity of the manufactured product. The measurement uncertainty previously assessed is inseparable from the measurement result and must be taken into account when concluding this declaration of conformity, or when determining the non-compliance.

The contractual requirement in a "customer-supplier" relationship may establish a prior agreement between the parties to set the limits of the value of the uncertainty.

To make decisions, the results are analyzed and 2 decision-making areas are taken into account, fig. 2 .

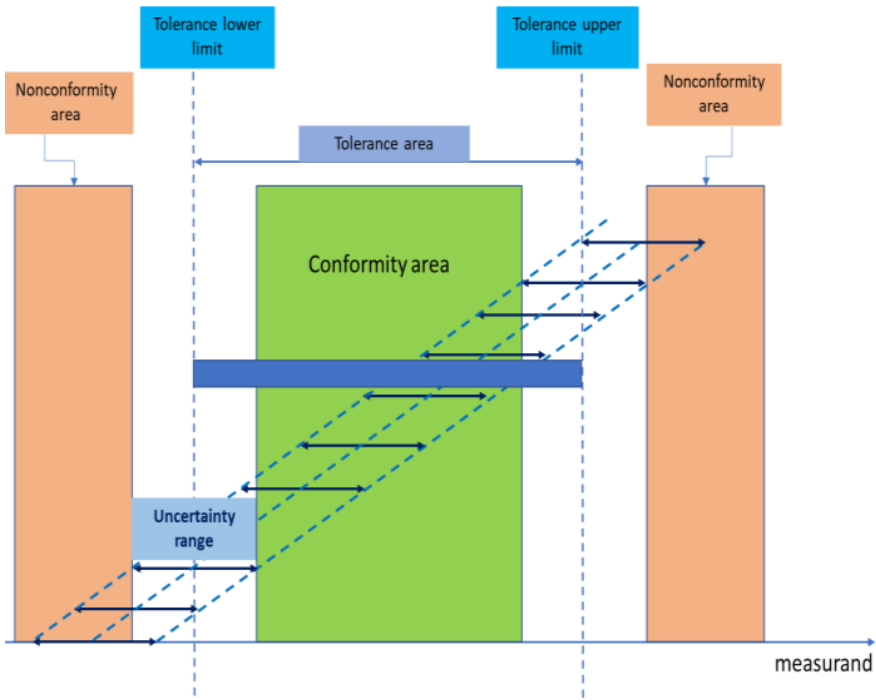

Figure 2. Interpretation of measurement results
Conformity area: the area within the specification with tolerances area minus the measurement uncertainty.

Non-compliance area: the area outside the specification area where the measurement uncertainty is gathered.

Therefore, the rules for making decisions are simple:

- The result of the measurement is within the conformity zone - the product is declared compliant;

- The measurement result is included in one of the non-conformity zones, the product is declared non-compliant; The result of the measurement is between the conformity zone and a non-compliance zone, no decision can be taken without a prior agreement between the 


\section{Experimental part}

\subsection{Dimensional measurements}

In a manufacturing workshop, parts are produced that in the documentation specified the diameter of $12,3 \pm 0,1 \mathrm{~mm}$; In order to ensure a very good quality of the manufacture, it was decided to consider the uncertainty when measuring the parts. There were achieved $\mathrm{N}=10$ diameter measurements were performed on the same part, by the same operator.

Table 1. Dimensional measurements

\begin{tabular}{|l|l|l|l|l|l|l|l|l|l|}
\hline$x_{1}$ & $x_{2}$ & $x_{3}$ & $x_{4}$ & $x_{5}$ & $x_{6}$ & $x_{7}$ & $x_{8}$ & $x_{9}$ & $x_{10}$ \\
\hline 12,2 & 12,28 & 12,31 & 12,39 & 12,4 & 12,35 & 12,23 & 12,26 & 12,37 & 12,33 \\
\hline
\end{tabular}

For the calculation we use the functions from the Excel program:

Standard deviation: $=\operatorname{STDEV}\left(x_{1}: x_{10}\right)=0,068280467$

$$
U_{\text {repet }}=0,068280467 / \sqrt{10}=0,02159203
$$

For a high 95\% reliability interval, we consider $\mathrm{k}=2, u=2 \cdot 0,02160337=0,043206742$

It turns out that we have a range of uncertainty $u=0,043 \mathrm{~mm}$.

\subsection{Vickers measurements}

In a testing laboratory they determine the hardness on some parts. The documentation specified the HV hardness 160-190. The contract with the client is specified that the laboratory must guaranty with a high confidence the quality of measured parts. The laboratory decided to consider the extended uncertainty, $\mathrm{k}=3$, for reliability interval $99,73 \%$. There were achieved $\mathrm{N}=17$ hardness, HV1. The measurements were performed on the same part, by the same operator.

Table 2. Vickers measurements

\begin{tabular}{|l|l|l|l|l|l|l|l|l|l|}
\hline$x_{1}$ & $x_{2}$ & $x_{3}$ & $x_{4}$ & $x_{5}$ & $x_{6}$ & $x_{7}$ & $x_{8}$ & $x_{9}$ & $x_{10}$ \\
\hline 180 & 181 & 172 & 168 & 169 & 165 & 168 & 161 & 165 & 167 \\
\hline
\end{tabular}

\begin{tabular}{|l|l|l|l|l|l|l|}
\hline$x_{11}$ & $x_{12}$ & $x_{13}$ & $x_{14}$ & $x_{15}$ & $x_{16}$ & $x_{17}$ \\
\hline 166 & 167 & 169 & 167 & 171 & 180 & 176 \\
\hline
\end{tabular}

Standard deviation $:=\operatorname{STDEV}\left(x_{1}: x_{17}\right)=5,84040188$

$$
\begin{aligned}
& S_{i=5,84040188} \\
& u_{\text {repet }}=5,84040188 / \sqrt{17}=1,41650552
\end{aligned}
$$

The laboratory use un certified Vickers tester; The tester uncertainty, estimated by metrological laboratory is $u_{\mathrm{t}}=4 \mathrm{HV} 1$

In this situation we calculate the combined uncertainty:

$$
u_{c}(x)=\sqrt{\sum_{i}\left(u_{i}(x)\right)^{2}}=4,24340522,
$$

$u_{\mathrm{i}}=$ uncertainties $\left(u_{\text {repet }}\right.$ and $\left.u_{t}\right)$

For a high $99,73 \%$ reliability interval, $u=3 * 4,24340522=12,7302157$

It turns out that we have a range of uncertainty $u=12,73 \mathrm{HV}$. 


\section{Results and discution}

\subsection{Dimensional measurement}

The graphical representation of the tolerance interval considering the uncertainty is presented in fig. 3 .

In this case, we can confidently estimate that there is a $\pm(0.043) / 2 \mathrm{~mm}$ uncertainty in the measurement. This is because a $12,2 \mathrm{~mm}$ measurement could really be anything from $12,18 \mathrm{~mm}$ (rounded up) to just under 12,22 $\mathrm{mm}$ (rounded down) and a 12,4 $\mathrm{mm}$ measurement could really be anything from $12,38 \mathrm{~mm}$ (rounded up) to just under 12,42 $\mathrm{mm}$ (rounded down). So, in order to be sure that the real value is in the tolerance range we have to restrict this interval with the value of the uncertainty, $\mathrm{u}$, in this example from 12,22 $\mathrm{mm}$ to $12,38 \mathrm{~mm}$.

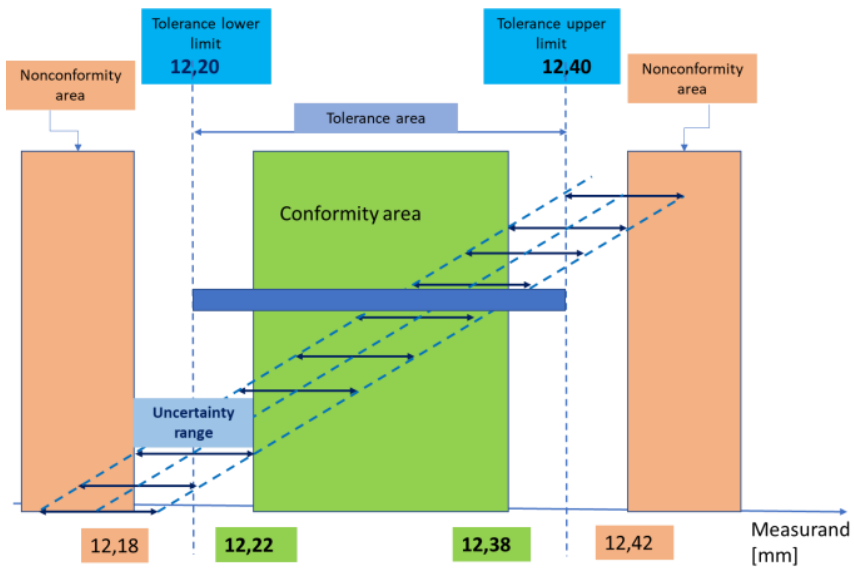

Figure 3. Graphical representation of the tolerance interval taking into account the uncertainty

It is observed that in order to ensure a level of reliability of $95 \%$ in the measurement results, only the pieces in the range of $12.22-12.38 \mathrm{~mm}$ will be considered compliant.

Table 3 The measurement results

\begin{tabular}{|l|l|l|l|l|l|l|l|l|l|}
\hline$x_{1}$ & $x_{2}$ & $x_{3}$ & $x_{4}$ & $x_{5}$ & $x_{6}$ & $x_{7}$ & $x_{8}$ & $x_{9}$ & $x_{10}$ \\
\hline 12,2 & 12,28 & 12,31 & 12,39 & 12,4 & 12,35 & 12,23 & 12,26 & 12,37 & 12,33 \\
\hline
\end{tabular}

In the analyzed example taking into account the measurement uncertainty 7 results are declared compliant with $95 \%$ confidence. We also find that 3 results, $\mathrm{x}_{1}, \mathrm{x}_{4}$ and $\mathrm{x}_{5}$ are outside this compliance range.

\subsection{Vickers measurements}

The graphical representation of the tolerance interval considering the uncertainty is presented in fig. 4.

In this case, we can confidently estimate that there is a $\pm(13,73) / 2 \mathrm{HV} 1$ uncertainty in the measurement. This is because a $160 \mathrm{HV} 1$ measurement could really be anything from $154 \mathrm{HV} 1$ (rounded up) to just under $166 \mathrm{HV} 1$ (rounded down) and a $190 \mathrm{HV} 1$ measurement could really be anything from 184 HV1 (rounded up) to just under $196 \mathrm{HV} 1$ (rounded down). So, in order to be sure that the real value is in the tolerance range, with $99,73 \%$ reliability, we have to restrict this interval with the value of the uncertainty, $u$, in this example from 166 to $184 \mathrm{HV} 1$.

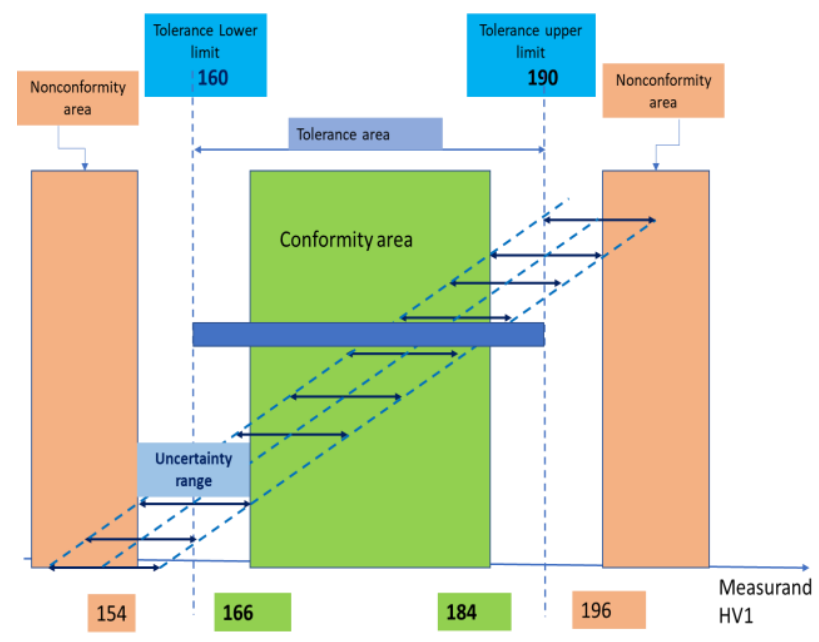

Figure 4. Graphical representation of the tolerance interval taking into account the uncertainty

It is observed, fig. 5, that in order to ensure a level of $99,73 \%$ reliability in the measurement results, only the measurements in the range of $166-184 \mathrm{~mm}$ will be considered compliant. In the analyzed 
example taking into account the measurement uncertainty 14 results are declared compliant with $99,73 \%$ confidence. We also find that 3 results are outside this compliance range.

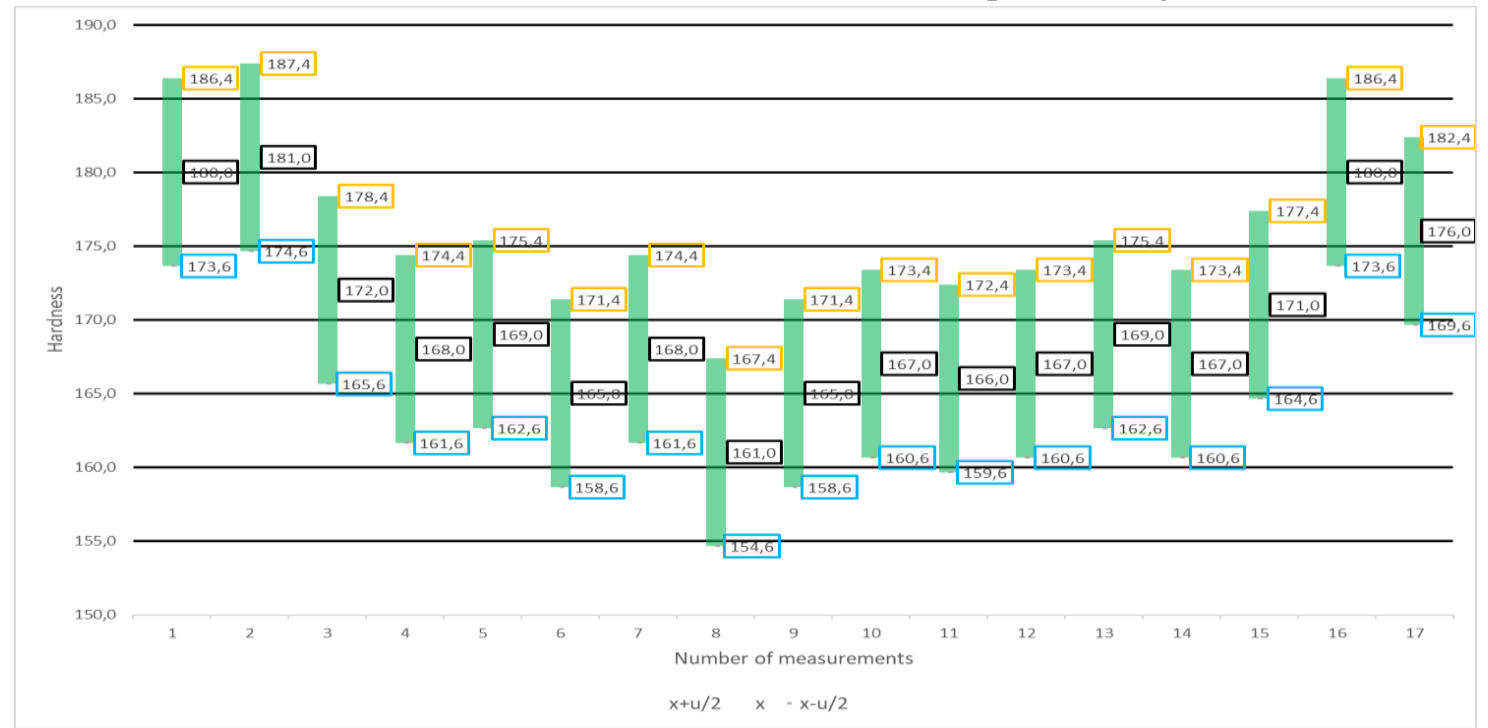

Figure 5. Graphical representation of Vickers measurements

\section{Conclusions}

Measurement uncertainty is an important parameter that contributes to increasing confidence in the measurement result, if the concept of uncertainty is used in the customer-supplier relationship or in measurement laboratories.

Measurement uncertainty is influenced by many factors, which were analyzed using the Cause-Effect diagram. Measurement uncertainty was determined based on several measurements using statistical analysis.

The graphical representation of the tolerance range taking into account the uncertainty also showed that the compliance area is reduced by the uncertainty value, thus increasing the confidence in the measurement results. The measurement uncertainty was calculated for a concrete example.

\section{References}

1. Mesure et incertitudes, Ministère de l'éducation nationale, de la jeunesse et de la vie associative (DGESCO-IGEN), mai 2012.

2. GUM (1995) Guide to the Expression of Uncertainty in Measurement. BIPM, IEC, IFCC, ISO,IUPAC, IUPAP, OIML. International Organization for Standardization, Switzerland, ISBN 9267-10188-9, Ist Edition, 1993. Corrected and reprinted 1995. On line at: www.bipm.org/en/publications/guides/gum.html

3. SR EN ISO CEI 17025 (2005) Cerinte generale pentru competenta laboratoarelor de încercări si etalonări.

4. EA 4/16 - EA guidelines on the expression of uncertainty in quantitative testing, https://europeanaccreditation.org/wp-content/uploads/2018/10/ea-4-02-m-rev01-september-2013.pdf

5. ISO 6507-1:2005 Metallic materials - Vickers hardness test - Part 1: Test method

6. ISO 6507-2:2005 Metallic materials - Vickers hardness test - Part 2: Verification and calibration of testing machines.

7. Emanuela BELDEAN, Uncertainty of measurement- an important instrument to evaluate the quality of results in formaldehyde tests, ISSN 2069-7430 ISSN-L 1841-4737, Vol. 9 Nr. 3 2013, pp. 51-59. 